\title{
Super-Luminal Effects for Finsler Branes as a Way to Preserve the Paradigm of Relativity Theories
}

\author{
Sergiu I. Vacaru* \\ Mathematical Physics Project IDEI, Alexandru Ioan Cuza University, UAIC, \\ Alexandru Lapuşneanu street, nr. 14, Corpus R, office 323; Iaşi, Romania, 700057
}

December 26, 2012

\begin{abstract}
Using Finsler brane solutions [see details and methods in: S. Vacaru, Class. Quant. Grav. 28 (2011) 215001], we show that neutrinos may surpass the speed of light in vacuum which can be explained by trapping effects from gravity theories on eight dimensional (co) tangent bundles on Lorentzian manifolds to spacetimes in general and special relativity. In nonholonomic variables, the bulk gravity is described by Finsler modifications depending on velocity/ momentum coordinates. Possible super-luminal phenomena are determined by the width of locally anisotropic brane (spacetime) and induced by generating functions and integration functions and constants in coefficients of metrics and nonlinear connections. We conclude that Finsler brane gravity trapping mechanism may explain neutrino super-luminal effects and almost preserve the paradigm of Einstein relativity as the standard one for particle physics and gravity.
\end{abstract}

Keywords: Super-luminal travel, Finsler branes, modified gravity, violation of local Lorentz symmetry.

PACS 2008: 04.50.Kd, 04.90.+e, 11.25.-w, 11.30.Cp, 02.40.-k

Introduction. It was a very surprising result in experimental particle physics that neutrino may travel faster than light in vacuum [1]. Perhaps, that experiment was not correct and there are not other ones confirming it correctness. Nevertheless, that generated in a series of papers 2] with different explanations implementing Lorentz symmetric violations, extra dimensions, quantum corrections from different string, noncommutative, nonlinear dispersion theories etc. If the metrics in such theories depend on

\footnotetext{
*sergiu.vacaru@uaic.ro, http://www.scribd.com/people/view/1455460-sergiu
} 
"velocity/momentum" type variables with warping/trapping, there are possible also super-luminal physical effects.

Classical and quantum physical theories with modifications of velocity/momentum constants and variables can be naturally modelled by modified dispersions and gravitational anisotropic polarizations of interaction constants and fields on (co) tangent bundles. Such nonlinear dispersion relations and related Finsler theories were studied in different approaches oriented to applications in modern cosmology, astrophysics and particle physics, or for elaborating generalized Finsler (super)string and noncommutative anisotropic gravity and nonholonomic Ricci flow theories, string/brane foam models etc [3, 4, 5, 6].

The goal of this letter is to show that super-luminal phenomena can be induced by trapping effects in Finsler gravity models [7] constructed on tangent bundles of Lorentzian manifolds for the general relativity theory (GR) and, in particular, when the base manifold is taken to be the Minkowski spacetime of the special relativity theory (SR). Finsler brane solutions with trapping to standard (four dimensional, 4-d) spacetime in GR and/or SR can be generated by diagonal and generic off-diagonal metrics in the bulk $(8-d)$ total space. Following this approach, we can perform a "canonical" Finsler generalization of axiomatics formulated for GR and SR which do not change the paradigm of standard relativity theories.

On Principles of Einstein-Finsler Gravity [8, 9]: Let us denote by $\mathbf{V}$ and, in particular, $M$, respectively, a 4-d Lorentz manifold (spacetime) and a Minkowski spacetime endowed with metrics $g_{i j}\left(x^{k}\right)$ and $\eta_{i j}$ of signature $(-+++)$. Any compatible with experimental data generalizations to locally anisotropic metrics $g_{i j}\left(x^{k}, y^{a}\right)$ depending on velocities $y^{a}$, or momenta $p_{a} 1$ should be defined by "small" modifications of geometric/physical objects from base spacetime manifold $\mathbf{V}$ to tangent bundle $T \mathbf{V}$ (in particular, on $T M)$. In total space, we shall label the local coordinates in the form $u=$ $(x, y)=\left\{u^{\alpha}=\left(x^{i}, y^{a}\right)\right\}$. We can introduce the so-called Finsler variables both on $\mathbf{V}$ and $T \mathbf{V}$ when up to frame/coordinate transforms

$$
\tilde{g}_{i j}\left(x^{k}, y^{a}\right) \sim \partial^{2} F^{2} / \partial y^{i} \partial y^{j}
$$

is determined by the non-degenerate Hessian of a generating (fundamental) Finsler function $F(x, y)$. Such a function (also called a Finsler metric) is usually chosen to be homogeneous on $y$-variables 2 and satisfies different

\footnotetext{
${ }^{1}$ by $x=\left(x^{i}\right)$ we denote local coordinates on $\mathbf{V}$ (indices $i, j, k \ldots$ and $a, b, c \ldots$ are coordinate or abstract ones which may take values $1,2,3,4$.

${ }^{2}$ various nonhomogeneous generating functions (for instance, regular Lagrangians) and
} 
conditions in "standard" Finsler geometry with signature $(++++)$, or for pseudo-Euclidean local signatures. For simplicity, we shall use the term Finsler for all possible signatures if that will not result in ambiguities, see details in Refs. [8, 9].

The nonlinear quadratic element $d s^{2}=F^{2}(x, y)$ in Finsler geometry generalizes the quadratic one in pseudo-Riemannian geometry, when $d s^{2}=$ $g_{i j}(x) y^{i} y^{j}$. In the second case, we can state $g_{i j}\left(x_{(0)}^{k}\right)=\eta_{i j}$ in any point $x_{(0)}^{i} \in \mathbf{V}$ and such a geometry is characterized by a unique torsionless and metric compatible connection $\nabla$ (the Levi-Civita connection). A spacetime in GR is determined by some data $\left(g_{i j}(x), \nabla(x)\right)$ as a solution of the Einstein gravitational field equations, $R_{i k}-\frac{1}{2} g_{i k} R=\varkappa T_{i k}$. In these formulas, $R_{i k}$ and $R$ are, respectively, the Ricci tensor and scalar curvature of $\nabla$ and $T_{i k}$ is the energy-momentum tensor for matter fields. The constant $\varkappa$ is determined by the Newton constant and the speed of light $c$ is taken to be constant in vacuum. An important property of the pseudo-Riemann geometry is that geodesics (i.e. extremals) of $g_{i j}$ are equivalent to auto-parallels of $\nabla$.

Prescribing a Finsler generating function $F(x, y)$, we do not define completely a geometric and/or physical model on $T \mathbf{V}$, see details and discussions in [8, 10]. Finsler spaces and related gravity models are with more rich geometric structures than the (pseudo) Riemann/ Lorentzian geometry. We need additional assumptions, following some geometric criteria and/or physical arguments, on classes of "admissible" total and base/fiber frames of references, metrics and linear connections. In brief, such considerations result in a statement that a Finsler geometry/gravity model is defined by data $(F: \mathbf{N}, \mathbf{g}, \mathbf{D})$ following certain conditions for three fundamental geometric objects (instead of one, $g_{i j}$, in GR):

1. $\mathbf{N}=\left\{\mathbf{N}_{i}^{a}(u)\right\}$ is a nonlinear connection ( $\mathrm{N}$-connection) which, up-to frame/coordinate transforms, is defined by semi-spray configurations, i.e. nonlinear geodesics which are equivalent to the Euler-Lagrange equations for the effective regular Lagrangian $L=F^{2}$. This states a nonholonomic (non-integrable) splitting $N: T T \mathbf{V}=h T \mathbf{V} \oplus v T \mathbf{V}$ into conventional horizontal $(\mathrm{h})$ and vertical (v) components, for an absolute (Whitney) sum $\oplus$. We can work in $\mathrm{N}$-adapted form on $T \mathbf{V}$ if there are used the so-called $\mathrm{N}$-elongated partial derivatives and differentials (equivalently, distinguished, d, frames and co-frames),

$$
\mathbf{e}_{\nu}=\left(\mathbf{e}_{i}=\partial_{i}-N_{i}^{a} \partial_{a}, e_{a}=\partial_{a}\right), \mathbf{e}^{\mu}=\left(e^{i}=d x^{i}, \mathbf{e}^{a}=d y^{a}+N_{i}^{a} d x^{i}\right) .
$$

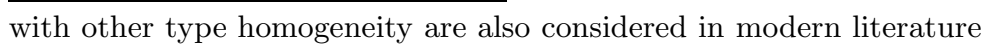


2. $\mathbf{g}=\left\{\mathbf{g}_{\alpha \beta}(u)\right\}$ is the metric in total space $T \mathbf{V}$ defined by $F$ and $\mathbf{N}$, usually via Sasaki lifts of $\tilde{g}_{i j}$ (1) and possible further frame/coordinate transforms to respective h- an v-metric, $g_{i j}$ and $h_{a b}$, when

$$
\mathbf{g}=g_{i j}(x, y) d x^{i} \otimes d x^{j}+h_{a b}(x, y) \mathbf{e}^{a} \otimes \mathbf{e}^{b}
$$

3. $\mathbf{D}$ is a distinguished connection ( $\mathrm{d}-$-connection), i.e. linear connection preserving under parallelism the $\mathrm{N}$-connection $\mathrm{h}-\mathrm{v}$-splitting. Realistic physical theories with Dirac operators and constructive-axiomatic approximations can be formulated for any metric compatible, $\mathbf{D g}=0$, when all values in distortion relation $\mathbf{D}=\nabla+\mathbf{Z}$, with distortion tensor $\mathbf{Z}$ being a combination of coefficients of torsion of $\mathbf{D}$, are completely defined by $\mathbf{g}$, which (in its turn) is induced by $F$, see details in [8, 10].

In general, Finsler geometries/theories are with metric non-compatible d-connections, $\mathbf{D g} \neq 0$. A typical example is that of Chern (also known as Rund) $d$-connection. Gravity and matter field models with metric noncompatible connection are less "relevant" to standard theories of particle physics because such locally anisotropic models do not allow "simple" definitions of spinors and Finsler-Dirac operators, conservation laws etc, see rigorous definitions and exact formulas for all geometric objects and critical remarks in [10] and references therein 3 To construct physically realistic models of Finsler classical and quantum gravity, locally anisotropic (non) commutative/ supersymmetric generalizations of string/brane/ nonholonomic Ricci flow theories etc is more convenient to use the Cartan $\mathrm{d}$-connection, $\mathbf{D}=\tilde{\mathbf{D}}$. This Finsler connection also defines a canonical almost Kähler structure and allows us to perform various types of deformation, A-brane and two connection quantization [11]. The canonical d-connection $\mathbf{D}=\hat{\mathbf{D}}$ is more convenient if we try to integrate the gravitational field equations in most general forms [9] and preserve similarity with the GR theory. We can construct certain physically important classes of exact solutions for $\hat{\mathbf{D}}$ and then to perform nonholonomic deformations to $\tilde{\mathbf{D}}$, or to impose nonholonomic constraints on $N_{i}^{a}$ when $\hat{\mathbf{D}}$ and $\nabla$ are described by the same sets of coefficients in $\mathrm{N}$-adapted frames (2).

An Einstein-Finsler gravity (EFG) model can be elaborated on $T \mathbf{V}$ following the same principles and axioms as for GR on $\mathbf{V}$ but, in our approach, for the data $(F: \mathbf{N}, \mathbf{g}, \hat{\mathbf{D}})$, see details in Refs. [8]. Here we note that the

\footnotetext{
${ }^{3}$ We consider that readers are familiar with basic concepts and constructions for the geometry of Lorentz manifolds and Finsler spaces; in this letter, we can not provide, explain and motivate all results and methods.
} 
data $(F: \mathbf{N}, \mathbf{g}, \tilde{\mathbf{D}})$ were used in [11], for quantum Finsler gravity models, and in [7, for Finsler brane solutions. For purposes of this work, it is enough to generalize the concept of Einstein manifold, when the gravitational field equations are of type $R_{i k}=\Lambda g_{i k}$, to that of Einstein-Finsler spaces determined by total metrics $\mathbf{g}_{\alpha \beta}$ as solutions of equations

$$
\hat{\mathbf{R}}_{\beta}^{\alpha}=\mathbf{\Upsilon}_{\beta}^{\alpha},
$$

where the source $\boldsymbol{\Upsilon}_{\beta}^{\alpha}(u)$ is generated via gravitational polarization of $\Lambda \delta^{\alpha}{ }_{\beta}$ (see below). In above formulas, the Ricci tensor $\hat{\mathbf{R}}_{\beta}^{\alpha}$ is constructed for $\hat{\mathbf{D}} ; \Lambda$ is a cosmological constant in the bulk $T \mathbf{V}$ and $\delta_{\beta}^{\alpha}$ is the Kronecker symbol.

Diagonal Solutions with Trapping for Finsler Branes. The simplest example of tangent bundle solution on $T M$ which defines a trivial Finsler brane configuration and trapping scenario with vanishing Nconnection structure is given by ansatz, $\mathbf{g}=$

$$
\phi^{2}\left(y^{5}\right) \eta_{i j} d x^{i} \otimes d x^{j}-\left(l_{P}\right)^{2} \bar{h}\left(y^{5}\right)\left[d y^{5} \otimes d y^{5}+d y^{6} \otimes d y^{6} \pm d y^{7} \otimes d y^{7} \pm d y^{8} \otimes d y^{8}\right],
$$

where $l_{P}$ is an effective analog Planck length (in the bulk $T \mathbf{V}$, one could be polarizations of physical constants, see [9, 7]), $\eta_{i j}=\operatorname{diag}[-1,1,1,1]$; $\alpha, \beta, \ldots=1,2,3,4$ and the fiber coordinates $y^{5}, y^{6}, y^{7}, y^{8}$ are velocity/ momentum type. This metric is a solution of EFG field equations (4) for sources

$$
\Upsilon_{\delta}^{\beta}=\Lambda-\stackrel{\circ}{M}^{-(m+2)} \bar{K}_{1}\left(y^{5}\right), \Upsilon^{5}{ }_{5}=\Upsilon_{6}^{6}=\Lambda-\stackrel{\circ}{ }^{-(m+2)} \bar{K}_{2}\left(y^{5}\right),
$$

where $\stackrel{\circ}{M}$ is a fundamental mass scale on $T \mathbf{V}$ and $m=2,3,4$ defines the number of non-compactified "extra" dimensions of velocity type if

$$
\phi^{2}\left(y^{5}\right)=\frac{3 \epsilon^{2}+a\left(y^{5}\right)^{2}}{3 \epsilon^{2}+\left(y^{5}\right)^{2}} \text { and } l_{P} \sqrt{\left|\bar{h}\left(y^{5}\right)\right|}=\frac{9 \epsilon^{4}}{\left[3 \epsilon^{2}+\left(y^{5}\right)^{2}\right]^{2}} \text {. }
$$

In above formulas, $a$ is an integration constant and the width of brane is $\epsilon$, with some fixed integration parameters when $\left.\frac{\partial^{2} \phi}{\partial\left(y^{5}\right)^{2}}\right|_{y^{5}=\epsilon}=0$ and $\left.l_{P} \sqrt{\left|\bar{h}\left(y^{5}\right)\right|}\right|_{y^{5}=0}=1$; this states the conditions that on diagonal branes the Minkowski metric on $T V$ is $6-\mathrm{d}$ or $8-\mathrm{d}$. The compatibility of such sources, field equations and conservation laws in Finsler spaces are studied in [7] (see there explicit formulas for $\bar{K}_{1}$ and $\bar{K}_{2}$ ). Such constructions for $m=2$ are similar to those for usual 6 - $\mathrm{d}$ diagonal brane solutions with trapping and other various warping mechanisms and nonlinear wave interactions [12. Nevertheless, there are substantial differences between Finsler branes and extra dimension branes. In our case, the width $\epsilon^{2}=40 M^{4} / 3 \Lambda$ is for a brane 
in a bulk with extra velocity/ momentum coordinates and certain constants are related to $l_{P}$; the variable $y^{5}$ has a finite maximal value $y_{0}^{5}$ on $T M$.

Off-Diagonal Solutions for Generic Finsler Branes. We construct a class of generic off-diagonal Finsler brane solutions for the canonical dconnection $\hat{\mathbf{D}}$. For such classes of locally anisotropic spacetimes, and via corresponding trapping mechanisms, there are possibilities to generate various classes of black hole, wormhole, cosmological metrics etc.

Let us consider a metric $\mathbf{g}=\left\{\mathbf{g}_{\alpha^{\prime} \beta^{\prime}}\right\}$ which via frame transforms on $T \mathbf{V}$, $\mathbf{g}_{\alpha^{\prime} \beta^{\prime}}=e_{\alpha^{\prime}}^{\alpha}(u) e_{\beta^{\prime}}^{\beta}(u) \widetilde{\mathbf{g}}_{\alpha \beta}$, can be transformed into a generic off-diagonal metric $\widetilde{\mathbf{g}}=\left\{\widetilde{\mathbf{g}}_{\alpha \beta}=\left[g_{i j}, h_{a b}\right]\right\}$. The ansatz for $\widetilde{\mathbf{g}}$ is taken in the form

$$
\begin{aligned}
\widetilde{\mathbf{g}}= & \phi^{2}\left(y^{5}\right)\left[g_{1} d x^{1} \otimes d x^{1}+g_{2} d x^{2} \otimes d x^{2}+h_{3} \mathbf{e}^{3} \otimes \mathbf{e}^{3}+h_{4} \mathbf{e}^{4} \otimes \mathbf{e}^{4}\right] \\
& +\left(l_{P}\right)^{2} \bar{h}\left(y^{5}\right)\left[h_{5} \mathbf{e}^{5} \otimes \mathbf{e}^{5}+h_{6} \mathbf{e}^{6} \otimes \mathbf{e}^{6}+h_{7} \mathbf{e}^{7} \otimes \mathbf{e}^{7}+h_{8} \mathbf{e}^{8} \otimes \mathbf{e}^{8}\right],
\end{aligned}
$$

where the coordinates are parametrized in the form $u^{\alpha}=\left(x^{1}, x^{2}, y^{3}=\right.$ $\left.v, y^{4}, y^{5}=\widetilde{v}, y^{6}, y^{7}=\underline{v}, y^{8}\right)$, the functions $\phi$ and $\bar{h}$ are those from (7) 4 and $e_{\alpha^{\prime}}^{\alpha}$ has nontrivial coefficients $e_{i^{\prime}}^{i}= \pm \phi \delta^{i}{ }_{i^{\prime}}$ and $e_{a^{\prime}}^{a}= \pm l_{P} \sqrt{\left|\bar{h}\left(y^{5}\right)\right|} \delta_{a^{\prime}}^{a}$. The metric $\widetilde{\mathbf{g}}$ is of type (3) which allows us to construct exact solutions of EFG field equations (44) following the anholonomic deformation method [9, 7]. In a more simple form, Finsler brane solutions can be generated for metrics with Killing symmetries on $\partial / y^{6}$ and $\partial / y^{8}$. In general, our method allows us to find solutions depending on all 8 coordinates but such constructions are very sophisticate and with less clear physical interpretation.

The coefficients of h-metric $g_{i j}=\left[g_{1}\left(x^{\widehat{k}}\right), g_{2}\left(x^{\widehat{k}}\right), h_{3}\left(x^{\widehat{k}}, v\right), h_{4}\left(x^{\widehat{k}}, v\right)\right]$, $\widehat{k}=1,2$, are parametrized with respect to a dual $\mathrm{N}$-adapted basis

$$
\begin{aligned}
\mathbf{e}^{i}= & \left(e^{1}=d x^{1}, e^{2}=d x^{2}, \mathbf{e}^{3}=d x^{3}+w_{1}\left(x^{\widehat{k}}, v\right) d x^{1}+w_{2}\left(x^{\widehat{k}}, v\right) d x^{2},\right. \\
& \left.\mathbf{e}^{4}=d x^{4}+n_{1}\left(x^{\widehat{k}}, v\right) d x^{1}+n_{2}\left(x^{\widehat{k}}, v\right) d x^{2}\right) .
\end{aligned}
$$

The coefficients of $\mathrm{v}-$ metric $h_{a b}=\left[h_{5}\left(x^{\widehat{k}}, \widetilde{v}\right), h_{6}\left(x^{\widehat{k}}, \widetilde{v}\right), h_{7}\left(x^{\widehat{k}}, \underline{v}\right), h_{8}\left(x^{\widehat{k}}, \underline{v}\right)\right]$ are parametrized with respect to

$$
\begin{aligned}
\mathbf{e}^{a} & =\left(\mathbf{e}^{5}=d y^{5}+\widetilde{w}_{\widehat{i}}\left(x^{\widehat{k}}, \widetilde{v}\right) d x^{\widehat{i}}, \mathbf{e}^{6}=d y^{6}+\widetilde{n}_{\widehat{i}}\left(x^{\widehat{k}}, \widetilde{v}\right) d x^{\widehat{i}},\right. \\
\mathbf{e}^{7} & \left.=d y^{7}+\underline{w}_{\hat{i}}\left(x^{\widehat{k}}, \underline{v}\right) d x^{\widehat{i}}, \mathbf{e}^{8}=d y^{8}+\underline{n}_{\hat{i}}\left(x^{\widehat{k}}, \underline{v}\right) d x^{\widehat{i}}\right) .
\end{aligned}
$$

The sources are any general ones which up to frame transforms can be written in $\mathrm{N}$-adapted form in a form $\boldsymbol{\Upsilon}^{\beta}{ }_{\delta}=$

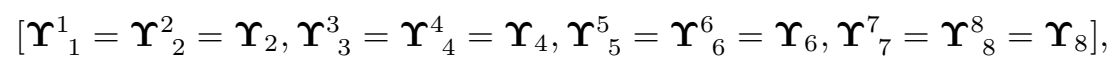

\footnotetext{
${ }^{4}$ for simplicity, we shall consider solutions with trapping on velocity type coordinate $y^{5}$, but the constructions can be performed in a similar for for other fiber variables
} 
with coefficients subjected to algebraic conditions when ${ }^{h} \Lambda\left(x^{\widehat{k}}\right)=\Upsilon_{4}+$ $\boldsymbol{\Upsilon}_{6}+\Upsilon_{8},{ }^{v} \Lambda\left(x^{\widehat{k}}, v\right)=\boldsymbol{\Upsilon}_{2}+\boldsymbol{\Upsilon}_{6}+\boldsymbol{\Upsilon}_{8},{ }^{5} \Lambda\left(x^{\widehat{k}}, \widetilde{v}\right)=\boldsymbol{\Upsilon}_{2}+\boldsymbol{\Upsilon}_{4}+\boldsymbol{\Upsilon}_{8},{ }^{7} \Lambda\left(x^{\widehat{k}}, \underline{v}\right)=$ $\boldsymbol{\Upsilon}_{2}+\boldsymbol{\Upsilon}_{4}+\boldsymbol{\Upsilon}_{6}$. For zero $\mathbf{N}$ - coefficients, such sources determine gravitational polarizations of "constant" cosmological constant and sources (6) ).

For data $(\widetilde{\mathbf{g}}, \hat{\mathbf{D}})$ corresponding to ansatz (8) , the nontrivial coefficients of the Ricci tensor $\widehat{R}_{\beta}^{\alpha}$ for the system (4) are computed [9, 7]

$$
\begin{aligned}
& \widehat{R}_{1}^{1}=\widehat{R}_{2}^{2}=\frac{1}{2 g_{1} g_{2}}\left[\frac{g_{1}^{\bullet} g_{2}^{\bullet}}{2 g_{1}}+\frac{\left(g_{2}^{\bullet}\right)^{2}}{2 g_{2}}-g_{2}^{\bullet \bullet}+\frac{g_{1}^{\prime} g_{2}^{\prime}}{2 g_{2}}+\frac{\left(g_{1}^{\prime}\right)^{2}}{2 g_{1}}-g_{1}^{\prime \prime}\right]=-{ }^{h} \Lambda, \\
& \widehat{R}_{3}^{3}=\widehat{R}_{4}^{4}=\frac{1}{2 h_{3} h_{4}}\left[-h_{4}^{* *}+\frac{\left(h_{4}^{*}\right)^{2}}{2 h_{4}}+\frac{h_{3}^{*} h_{4}^{*}}{2 h_{3}}\right]=-{ }^{v} \Lambda, \\
& \widehat{R}_{5}^{5}=\widehat{R}_{6}^{6}=\frac{1}{2 h_{5} h_{6}}\left[-h_{6}^{\circ \circ}+\frac{\left(h_{6}^{\circ}\right)^{2}}{2 h_{6}}+\frac{h_{5}^{\circ} h_{6}^{\circ}}{2 h_{5}}\right]=-{ }^{5} \Lambda, \\
& \widehat{R}_{7}^{7}=\widehat{R}_{8}^{8}=\frac{1}{2 h_{7} h_{8}}\left[-h_{8}^{\diamond \diamond}+\frac{\left(h_{8}^{\diamond}\right)^{2}}{2 h_{8}}+\frac{h_{7}^{\diamond} h_{8}^{\diamond}}{2 h_{7}}\right]=-{ }^{7} \Lambda, \\
& 2 h_{4} \widehat{R}_{3 \widehat{k}}=-w_{\widehat{k}}\left[-h_{4}^{* *}+\frac{\left(h_{4}^{*}\right)^{2}}{2 h_{4}}-\frac{h_{3}^{*} h_{4}^{*}}{2 h_{3}}\right]+\partial_{\widehat{k}}\left(h_{4}^{*}-\ln \sqrt{\left|h_{3} h_{4}\right|}\right)=0, \\
& 2 \frac{h_{3}}{h_{4}} \widehat{R}_{4 \widehat{k}}=n_{\widehat{k}}^{* *}+\left(2 h_{3}^{*}-3 \frac{h_{4}^{*}}{h_{4}}\right) n_{\widehat{k}}^{*}=0, \\
& 2 h_{6} \widehat{R}_{5 \widehat{k}}=-\widetilde{w}_{\widehat{k}}\left[-h_{6}^{\circ}+\frac{\left(h_{6}^{\circ}\right)^{2}}{2 h_{6}}+\frac{h_{5}^{\circ} h_{6}^{\circ}}{2 h_{5}}\right]-\partial_{\widehat{k}}\left(h_{6}^{\circ}-\ln \sqrt{\left|h_{5} h_{6}\right|}\right)=0, \\
& 2 \frac{h_{5}}{h_{6}} \widehat{R}_{6 \widehat{k}}=\widetilde{n}_{\widehat{k}}^{\circ \circ}+\left(2 h_{5}^{\circ}-3 \frac{h_{6}^{\circ}}{h_{6}}\right) \widetilde{n}_{\widehat{k}}^{\circ}=0, \\
& 2 h_{8} \widehat{R}_{7 \widehat{k}}=-\underline{w}_{\widehat{k}}\left[-h_{8}^{\diamond \diamond}+\frac{\left(h_{8}^{\diamond}\right)^{2}}{2 h_{8}}+\frac{h_{7}^{\diamond} h_{8}^{\diamond}}{2 h_{7}}\right]-\partial_{\widehat{k}}\left(h_{8}^{\diamond}-\ln \sqrt{\left|h_{7} h_{8}\right|}\right)=0, \\
& 2 \frac{h_{7}}{h_{8}} \widehat{R}_{8 \widehat{k}}=\underline{n}_{\widehat{k}}^{\diamond \diamond}+\left(2 h_{7}^{\diamond}-3 \frac{h_{8}^{\diamond}}{h_{8}}\right) \underline{n}_{\widehat{k}}^{\diamond}=0,
\end{aligned}
$$

where $\widehat{k}=1,2$ and certain partial derivatives are denoted in a "brief" form, $g_{1}^{\bullet} \equiv \partial g_{1} / \partial x^{1}, g_{1}^{\prime} \equiv \partial g_{1} / \partial x^{2}, h_{4}^{*} \equiv \partial h_{4} / \partial v, h_{5}^{\circ} \equiv \partial h_{5} / \partial \widetilde{v}, h_{7}^{\diamond} \equiv \partial h_{7} / \partial \underline{v}$.

The above system of equations (10)-(19) reflects an important decoupling property, with respect to $\mathrm{N}$-adapted frames, of field equations for different classes of gravitational theories 5 For instance, the equation (10) is for a diagonal metric on a $2-\mathrm{d}$ subspace. The equation (11) contains only the first and second derivative on $*=\partial / \partial v$. Prescribing, for instance, $h_{4}\left(x^{\widehat{k}}, v\right)$ and ${ }^{v} \Lambda\left(x^{\widehat{k}}, v\right)$, we can almost always integrate on $d v$ and find $h_{3}\left(x^{\widehat{k}}, v\right)$ up to

\footnotetext{
${ }^{5}$ in our works on GR and noncommutative, metric-affine, supersymmetric, string, brane, generalized Finsler etc extensions 13, 9, 7, 8, we provided examples when the fundamental equations (4), split into certain sub-systems of nonlinear partial differential equations (NPDE) which can be integrated in very general forms.
} 
certain classes of generating functions, integration functions and constants and certain parameters. Having defined both $h_{3}$ and $h_{4}$, for "well-defined" conditions, we can can solve the algebraic equations (14) for $w_{\widehat{k}}\left(x^{\widehat{k}}, v\right)$ and (after two integrations on $d v$ ) the second order PDE (15), in order to find $n_{\widehat{k}}\left(x^{\widehat{k}}, v\right)$. Here we note that in a similar form we can construct solutions of systems (12), (16) and (17) (and, respectively, of (13), (18) and (19)) when $*=\partial / \partial v$ is changed into $\circ=\partial / \partial \widetilde{v}$ (and, respectively, $\diamond=\partial / \partial \underline{v}$ ) for corresponding coefficients of (8) .

We show how the EFG equations can be integrated in very general forms for ansatz (8) with $h_{4}^{*} \neq 0, h_{6}^{\circ} \neq 0$ and $h_{6}^{\diamond} \neq 0$. Introducing functions

$$
\begin{aligned}
g_{\widehat{k}} & =\epsilon_{\widehat{k}} \exp \psi\left(x^{\widehat{\hat{i}}}\right), \epsilon_{\widehat{k}}= \pm 1, \quad \text { depending on signature, } \\
\phi & :=\ln \mid h_{4}^{*} / \sqrt{\left|h_{3} h_{4}\right|}, \gamma:=\left[\ln \left(\left|h_{4}\right|^{3 / 4} /\left|h_{3}\right|\right)\right]^{*}, \alpha_{\widehat{k}}=h_{4}^{*} \partial_{\widehat{k}} \phi, \beta=h_{4}^{*} \phi^{*}, \\
\widetilde{\phi} & :=\ln \mid h_{6}^{\circ} / \sqrt{\left|h_{5} h_{6}\right|}, \widetilde{\gamma}:=\left[\ln \left(\left|h_{6}\right|^{3 / 4} /\left|h_{5}\right|\right)\right]^{\circ}, \widetilde{\alpha}_{\widehat{k}}=h_{6}^{\circ} \partial_{\widehat{k}} \widetilde{\phi}, \widetilde{\beta}=h_{6}^{\circ} \widetilde{\phi}^{\circ}, \\
\underline{\phi} & :=\ln \left|h_{8}^{\diamond} / \sqrt{\left|h_{7} h_{8}\right|}\right|, \underline{\gamma}:=\left[\ln \left(\left|h_{8}\right|^{3 / 4} /\left|h_{7}\right|\right)\right]^{\curvearrowright}, \underline{\alpha}_{\widehat{k}}=h_{8}^{\diamond} \partial_{\widehat{k}} \underline{\phi}, \underline{\beta}=h_{8}^{\diamond} \underline{\phi}^{\diamond} ;
\end{aligned}
$$

for $\phi^{*} \neq 0, \widetilde{\phi}^{\circ} \neq 0, \underline{\phi}^{\diamond} \neq 0$; in (10)-(19), we get $\epsilon_{1} \psi^{\bullet \bullet}+\epsilon_{2} \psi^{\prime \prime}=2^{h} \Lambda$,

$$
\begin{aligned}
& \phi^{*} h_{4}^{*}=2 h_{3} h_{4}{ }^{v} \Lambda, \beta w_{\widehat{k}}+\alpha_{\widehat{k}}=0, n_{\widehat{i}}^{* *}+\gamma n_{\widehat{i}}^{*}=0, \\
& \widetilde{\phi}^{\circ} h_{6}^{\circ}=2 h_{5} h_{6}{ }^{5} \Lambda, \widetilde{\beta} \widetilde{w}_{\widehat{k}}+\widetilde{\alpha}_{\widehat{k}}=0, \widetilde{n}_{\widehat{i}}^{\circ}+\widetilde{\gamma} \widetilde{n}_{\hat{i}}^{\circ}=0, \\
& \underline{\phi}^{\diamond} h_{8}^{\diamond}=2 h_{7} h_{8}{ }^{7} \Lambda, \underline{\beta} \underline{w}_{\widehat{k}}+\underline{\alpha}_{\widehat{k}}=0, \underline{n}_{\hat{i}}^{\diamond}+\underline{\gamma}_{\widehat{i}}^{\diamond}=0 .
\end{aligned}
$$

This system of equations can be integrated "step by step" when, finally, the most important coefficients are expressed

$$
\begin{aligned}
& h_{4}= \pm \frac{1}{4} \int d v\left|{ }^{v} \Lambda\right|^{-1}\left(e^{2 \phi}\right)^{*}, \text { or }= \pm \frac{1}{4^{v} \Lambda} e^{2\left[\phi-{ }^{0} \phi\left(x^{\widehat{k}}\right)\right]}, \text { if }{ }^{v} \Lambda=\text { const; } \quad(20) \\
& h_{3}= \pm\left[\left(\sqrt{\left|h_{4}\right|}\right)^{*}\right]^{2} e^{-2 \phi}=\frac{\phi^{*}}{\left.2\right|^{v} \Lambda \mid}\left(\ln \left|h_{4}\right|\right)^{*}, \text { or }= \pm \frac{\left(\phi^{*}\right)^{2}}{4^{v} \Lambda}, \text { if }{ }^{v} \Lambda=\text { const; } \\
& w_{\widehat{i}}=-\partial_{\hat{i}} \phi / \phi^{*}, n_{\widehat{i}}={ }^{1} n_{\widehat{i}}\left(x^{\widehat{k}}\right)+{ }^{2} n_{\widehat{i}}\left(x^{\widehat{k}}\right) \int d v h_{3}\left|h_{4}\right|^{-3 / 2}, \\
& h_{6}= \pm \frac{1}{4} \int d \widetilde{v}\left|{ }^{5} \Lambda\right|^{-1}\left(e^{2 \widehat{\phi}}\right)^{*}, \text { or }= \pm \frac{1}{4^{5} \Lambda} e^{2\left[\widetilde{\phi}-{ }^{0} \widetilde{\phi}\left(x^{\widehat{k}}\right)\right]}, \text { if }{ }^{5} \Lambda=\text { const; } \\
& h_{5}= \pm\left[\left(\sqrt{\left|h_{6}\right|}\right)^{\circ}\right]^{2} e^{-2 \widetilde{\phi}}=\frac{\phi^{\circ}}{\left.2\right|^{5} \Lambda \mid}\left(\ln \left|h_{6}\right|\right)^{\circ}, \text { or }= \pm \frac{\left(\phi^{\circ}\right)^{2}}{4^{5} \Lambda}, \text { if }{ }^{5} \Lambda=\text { const; } \\
& \widetilde{w}_{\widehat{i}}=-\partial_{\widehat{i}} \widetilde{\phi} / \widetilde{\phi}^{\circ}, \widetilde{n}_{\widehat{i}}={ }^{1} \widetilde{n}_{\widehat{i}}\left(x^{\widehat{k}}\right)+{ }^{2} \widetilde{n}_{\widehat{i}}\left(x^{\widehat{k}}\right) \int d \widetilde{v} h_{5}\left|h_{6}\right|^{-3 / 2},
\end{aligned}
$$




$$
\begin{aligned}
& h_{8}= \pm \frac{1}{4} \int d \underline{v}\left|{ }^{7} \Lambda\right|^{-1}\left(e^{2 \underline{\phi}}\right)^{\diamond}, \text { or }= \pm \frac{1}{4^{7} \Lambda} e^{2\left[\underline{\underline{\phi}}-{ }^{0} \underline{\phi}\left(x^{\widehat{k}}\right)\right]}, \text { if }{ }^{7} \Lambda=\mathrm{const} ; \\
& h_{7}= \pm\left[\left(\sqrt{\left|h_{8}\right|}\right)^{\diamond}\right]^{2} e^{-2 \underline{\phi}}=\frac{\phi^{\diamond}}{2\left|{ }^{7} \Lambda\right|}\left(\ln \left|h_{8}\right|\right)^{\diamond}, \text { or }= \pm \frac{\left(\phi^{\diamond}\right)^{2}}{4^{7} \Lambda}, \text { if }{ }^{7} \Lambda=\mathrm{const} ; \\
& \underline{w}_{\widehat{i}}=-\partial_{\hat{i}} \underline{\phi} / \underline{\phi}^{\diamond}, \underline{n}_{\widehat{i}}={ }^{1} \underline{\underline{n}}_{\widehat{i}}\left(x^{\widehat{k}}\right)+{ }^{2} \underline{\underline{n}}_{\hat{i}}\left(x^{\widehat{k}}\right) \int d \underline{v} h_{7}\left|h_{8}\right|^{-3 / 2} .
\end{aligned}
$$

Above presented classes of generic off-diagonal solutions depend respectively on generating functions $\psi\left(x^{\widehat{k}}\right), \phi\left(x^{\widehat{k}}, v\right), \widetilde{\phi}\left(x^{\widehat{k}}, \widetilde{v}\right), \underline{\phi}\left(x^{\widehat{k}}, \underline{v}\right)$ and on integration functions/constants ${ }^{0} \phi\left(x^{\widehat{k}}\right), \quad{ }^{0} \widetilde{\phi}\left(x^{\widehat{k}}\right),{ }^{0} \underline{\phi}\left(x^{\widehat{k}}\right),{ }^{1} n_{\widehat{i}}\left(x^{\widehat{k}}\right),{ }^{2} n_{\widehat{i}}\left(x^{\widehat{k}}\right)$, ${ }^{1} \widetilde{n}_{\widehat{i}}\left(x^{\widehat{k}}\right),{ }^{2} \widetilde{n}_{\widehat{i}}\left(x^{\widehat{k}}\right),{ }^{1} \underline{n}_{\hat{i}}\left(x^{\widehat{k}}\right),{ }^{2} \underline{n}_{\hat{i}}\left(x^{\widehat{k}}\right)$ etc. Such values should be defined in explicit form from certain physical considerations by imposing additional symmetries (for instance, ellipsoid/torus configurations, solitons etc), boundary conditions, Cauchy problem etc. In a more general context, the solutions may depend on constant commutative and noncommutative parameters, nonlinear solitonic hierarchies etc, see examples in Ref. [9]. It should be noted that the limit $\Lambda \rightarrow 0$ may be not smooth for certain classes of solutions. As particular cases, such metrics and generalize Finsler and other type connections describe deformations from black hole, wormhole, cosmological metric in GR and various modifications (we can use examples from [9, 7, 8, 10, 13] etc).

The (generalized) Finsler configurations determined by any nontrivial (20), (21) etc are positively with nontrivial torsion induced completely by the metric structure (8) via corresponding nonhlonomic effect of $\mathrm{N}$-connection and anholonomic $\mathrm{N}$-adapted frames. This is the property of the canonical $\mathrm{d}$-connection $\hat{\mathbf{D}}$ (and of the Cartan $\mathrm{d}$-connection $\tilde{\mathbf{D}}$ ). Imposing additional nonholonomic constraints

$$
\begin{aligned}
w_{\hat{i}}^{*} & =\left(\partial_{\hat{i}}-w_{\widehat{i}}\right) \ln \left|h_{4}\right|, \partial_{\widehat{k}} w_{\widehat{i}}=\partial_{\hat{i}} w_{\widehat{k}}, \partial_{\widehat{i}} n_{\widehat{k}}=\partial_{\widehat{k}} n_{\widehat{i}}, \\
\widetilde{w}_{\hat{i}}^{\circ} & =\left(\partial_{\widehat{i}}-\widetilde{w}_{\hat{i}}\right) \ln \left|h_{6}\right|, \partial_{\widehat{k}} \widetilde{w}_{\hat{i}}=\partial_{\hat{i}} \widetilde{w}_{\widehat{k}}, \partial_{\hat{i}} \widetilde{n}_{\widehat{k}}=\partial_{\widehat{k}} \widetilde{n}_{\hat{i}}, \\
\underline{w}_{\hat{i}}^{\diamond} & =\left(\partial_{\hat{i}}-\underline{w}_{\hat{i}}\right) \ln \left|h_{8}\right|, \partial_{\widehat{k}} \underline{w}_{\hat{i}}=\partial_{\hat{i}} \underline{w}_{\widehat{k}}, \partial_{\hat{i}} \underline{n}_{\widehat{k}}=\partial_{\widehat{k}} \underline{n}_{\widehat{i}},
\end{aligned}
$$

we can generate EFG solutions with zero torsion on $T \mathbf{V}$ which describe models of GR extended on its tangent bundle to Lorentz manifolds. If the so-called Levi-Civita conditions (22) are satisfied, the integration functions ${ }^{2} n_{\widehat{i}}\left(x^{\widehat{k}}\right),{ }^{2} \widetilde{n}_{\widehat{i}}\left(x^{\widehat{k}}\right), \quad{ }^{2} \underline{n}_{\hat{i}}\left(x^{\widehat{k}}\right)$ must be fixed zero. This allows us, in principle, to distinguish by future experiments if super-luminal and other effects are on generic Finsler branes (with nontrivial torsion) or on extensions on $T \mathbf{V}$ with the Levi-Civita connection $\nabla$. 
Effective Super-Luminal Speeds and Modified Dispersions. Let us show explicitly that trapping from Finsler branes may result in superluminal effects in real physical spacetime. Light rays in SR can be parametrized as $x^{i}(\varsigma)$ with a real smooth parameter $0 \leq \varsigma \leq \varsigma_{0}$, when $d s^{2} / d \varsigma^{2}=0$. We can consider a "null" tangent vector field $y^{i}(\varsigma)=d x^{i} / d \varsigma$, with $d \tau=$ $d t / d \varsigma$. Under general coordinate transforms $x^{i^{\prime}}=x^{i^{\prime}}\left(x^{i}\right)$, we have $\eta_{i j} \rightarrow$ $g_{i^{\prime} j^{\prime}}\left(x^{k}\right)$. The condition $d s^{2} / d \varsigma^{2}=0$ holds always for propagation of light, i.e. $g_{i^{\prime} j^{\prime}} y^{i^{\prime}} y^{j^{\prime}}=0$. The Minkowski metric $\eta_{i j}=\operatorname{diag}[-1,+1,+1,+1]($ for $i=$ $1,2,3,4)$ determines the quadratic line element $d s^{2}=\eta_{i j} d x^{i} d x^{j}=-\left(d x^{1}\right)^{2}+$ $\left(d x^{2}\right)^{2}+\left(d x^{3}\right)^{2}+\left(d x^{4}\right)^{2}$, with space type, $\left(x^{2}, x^{3}, x^{4}\right)$, and time like, $x^{1}=c t$, coordinates where $c$ is the constant speed of light (in vacuum). We use indices of type $\bar{i}, \bar{j}, \ldots=2,3,4$, when $c^{2}=\widetilde{g}_{\overline{i j}}\left(x^{i}\right) y^{\bar{i}} y^{\bar{j}} / \tau^{2}$. This formula can be considered in GR if $g_{\widehat{i} \hat{j}}\left(x^{i}\right)$ are solutions of Einstein equations. In EFG, such a formula is generalized to

$$
\widetilde{c}^{2}\left(u^{\alpha}\right)=\widetilde{g}_{\overline{i j}}\left(u^{\alpha}\right) y^{\bar{i}} y^{\bar{j}} / \tau^{2},
$$

where $\widetilde{g}_{\overline{i j}}$ is determined by the h-component of solution of (4). Effective super-luminal speeds can be "obtained" from diagonal solutions (5) with $\phi^{2}(\tilde{v})=1+(a-1)(\tilde{v})^{2} /\left[3 \epsilon^{2}+(\tilde{v})^{2}\right]$, see formula (7). Here we do not consider fiber contributions in the total metric of order $\left(l_{P}\right)^{2}$ and $\epsilon^{4}$ which are of order of Planck length and with very small modifications for thick branes. Considering that $\widetilde{g}_{\overline{i j}}\left(u^{\alpha}\right) \simeq \phi^{2} \eta_{\overline{i j}}$, we express (23) in the form

$$
\widetilde{c}^{2}(a, \tilde{v}, \epsilon)=c^{2}+\triangle=c^{2}\left(1+\frac{(a-1)(\tilde{v})^{2}}{3 \epsilon^{2}+(\tilde{v})^{2}}\right)
$$

which mean that we get super-luminal speeds if $\triangle / c^{2}>0$, when the integration constat $a>1$. So, if the OPERA experimental data are correct, they may be explained as a trapping from diagonal solutions from the bulk of a brane configuration on $T M$.

Experiments with super-luminal effects can be applied to study possible locally anisotropic generalizations of spacetime structure to $T \mathbf{V}$. For instance, introducing the $\mathrm{h}$-component of a generic off-diagonal solution (8) with coefficients (20) and (21) into (23) we can compute locally anisotropic oscillations, any possible fractional and fractal spacetime structure, quantum fluctuations, or limits to modified (increases and/or decreased) maximal values of effective speed of light.

The above quadratic on $y^{\bar{i}}$ expressions can be re-written for arbitrary nonlinear ones, $\check{F}^{2}\left(x^{k}, y^{\bar{j}}\right)$. This way, we can model propagation of light in 
anisotropic media and/or construct an (aether) spacetime geometry when the 8-d bulk is derived from a 4-d Lorenzian manifold. For such constructions, we can use nonlinear homogeneous quadratic elements,

$$
d s^{2}=F^{2}\left(x^{i}, y^{j}\right) \approx-(c d t)^{2}+g_{\overline{i j}}\left(x^{k}\right) y^{\bar{i}} y^{\bar{j}}\left[1+\frac{1}{r} \frac{q_{\bar{i}_{1} \bar{i}_{2} \ldots \bar{i}_{2 r}}\left(x^{k}\right) y^{\bar{i}_{1}} \ldots y^{\bar{i}_{2 r}}}{\left(g_{\overline{i j}}\left(x^{k}\right) y^{\bar{i}} y^{\bar{j}}\right)^{r}}\right]+O\left(q^{2}\right),
$$

when $F\left(x^{i}, \beta y^{j}\right)=\beta F\left(x^{i}, y^{j}\right)$, for any $\beta>0$. It is possible to write such a relation in a fixed point $x^{k}=x_{(0)}^{k}$, when $g_{\overline{i j}}\left(x_{0}^{k}\right)=g_{\overline{i j}}$ and $q_{\bar{i}_{1} \bar{i}_{2} \ldots \bar{i}_{2 r}}=q_{\bar{i}_{1} \bar{i}_{2} \ldots \bar{i}_{2 r}}$ $\left(x_{0}^{k}\right)$. A nonlinear element (24) defines a nonlinear modified dispersion relation (MDR) between the frequency $\omega$ and the wave vector $k_{i}$ of light waves,

$$
\omega^{2}=c^{2}\left[g_{\overline{i j}} k^{\bar{i}} k^{\bar{j}}\right]^{2}\left(1-\frac{1}{r} \frac{q_{\bar{i}_{1} \bar{i}_{2} \ldots \bar{i}_{2 r}} k^{\bar{i}_{1}} \ldots k^{\bar{i}_{2 r}}}{\left[g_{\overline{i j}} k^{\bar{i}} k^{\bar{j}}\right]^{2 r}}\right) .
$$

As a matter of principe, we can associate an effective nonlinear Finsler metric to any such MDR which model violations of local Lorenz invariance (LV) when coefficients $q_{\bar{i}_{1}} \bar{i}_{2} \ldots \bar{i}_{2 r}$ are computed for different quantum gravity (QG) phenomenology models, or (in this work) for super-luminal phenomena via solutions of EFG equations.

Let us discuss some important issues on MDR, Finsler theories and modified theories of gravity and possible implications in explanation of possible super-luminal and QG effects. In [5], a theoretical study of existing experimental data was performed. Authors' conclusion was that the coefficients $q_{\bar{i}_{1} \bar{i}_{2} \ldots \bar{i}_{2 r}}$ in (24) and related (25) seem to be very small and this sounds to be very pessimistic for detecting a respective QG phenomenology and LV. Following such considerations, we may consider that Finsler brane superluminal effects are in contradiction to those results.

In [7], we emphasized that local considerations based on MDR with kinematic effects for a Finsler metric $F(x, y)$ are necessary and very important to study possible LV (and, in this work, super-luminal) propagation of particles and classical and quantum interaction. But any such way derived conclusion is not an complete one because parametrizations (24) are "geometric gauge" dependent. Using frame/coordinate transforms and nonholonomic deformations, $(F: \mathbf{g}, \mathbf{N}, \mathbf{D}) \rightarrow\left({ }^{0} F: \check{\mathbf{g}}, \check{\mathbf{N}}, \check{\mathbf{D}}\right)$, when ${ }^{0} F$ is a typical quadratic form in GR, possible LV and super-luminal effects are removed into data $(\check{\mathbf{N}}, \check{\mathbf{D}})$ modeling nonlinear generic off-diagonal quantum, and quasi-classical, interactions in QG.

In the bulk 8-d spacetime, the solutions of EFG are with locally anisotropic gravitational interactions and broken spacetime symmetries but on the 
base spacetime manifolds, with respect to $\mathrm{N}$-adapted frame of references, the MDR (25) may not reflect such properties. We constructed in explicit form such classes of solutions of noncommutative Finsler black holes [4], see also references therein. Physical effects for black hole/ellipsod, Taub NUT and wormhole solutions and various gravitational-gauge-fermion interactions (in GR, extra dimension gravity, and EFG, see [13) can not be studied experimentally only via Mikelson-Morley and related MDR determined by $F$ without further assumptions on Finsler connections and fundamental field equations. Exact solutions with generic off-diagonal metrics and anholonomic frames (nonlinear connection) and induced torsion effects effects are of crucial importance in Finsler theories and velocity/momentum variables.

Different classes of solutions depend on the type of Finsler connections, symmetry configurations, boundary conditions etc. Nevertheless, it is possible to conclude following general arguments and main properties of locally anisotropic theories if possible extensions of GR on $T \mathbf{V}$ are of Finsler type, or of Kaluza-Klein (with certain compactifications of velocity/momentum coordinates), or a variant of "velocity" extra dimension Einstein theory (with zero torsion). For generic Finsler configurations, the solutions for $n_{\hat{i}}$ coefficients depend on velocity. If the tangent bundle spacetime theory is of another type, it will be not such a dependence, or it will be not detected for some values higher then the limit of "compactification" on $v$-coordinates. Sure, super-luminal effects may exist in vicinity of black holes, for cosmological metrics with locally anisotropic generalizations etc. Physically important generic off-diagonal metrics of type (8), and more general nonKilling configurations, should contain in certain corresponding limits black hole, homogeneous and nonhomogeneous cosmological solutions or other metrics. Such solutions can constructed following methods elaborated in Refs. [9, 7, 8, 10, 13].

Concluding Remarks and Further Perspectives. This paper was inspired by results about possible superluminal propagation of neutrinos (additionally to the recent OPERA result [1], there were reported such data by FERMILAB07-MINO, FERMILAB79 collaborations [15]). At present, it is considered that those experiments were performed not correctly. Nevertheless, we prove theoretically that superluminality effects may exist in a total Finsler like bulk even the principles of relativity are correct and our physical spacetime is a Lorentz base manifold.

In our approach, we do not suggest violation of the Poincaré invariance at the fundamental level even we addressed to Finsler spacetime geometry 
models, which are naturally related to modified dispersion relations (MDR) and violation of local Lorentz invariance (LV). Such "radical" possibilities with changing paradigms of spectial relativity (SR) and general relativity (GR), violation of metric compatibility etc, were considered in Refs. [14, see also a series of articles [3]. The goal of this paper is to ask and provide an answer to the question: what minimal changes to the standard models of physics could explain superluminal phenomena and how this can be accommodated to existing principles and axiomatics of Einstein relativity theories?

Any constructions related to modifications for a maximal speed of interactions which do not change drastically the existing paradigms should be performed for (co) tangent bundles to Lorentz and Minkowski manifolds (respectively, in GR and SR) and this should be related to certain small MDR. Logically, we suppose that fundamental principles are still valid on Einstein spacetimes as brane configurations imbedded into (co) tangent bundle bulk generalizations, with finite effective velocities and polarizations of constants and field interactions. Such models can be always described effectively in so-called Finsler variables. Nonholonomic variables of Finsler and other type equivalent ones can be introduced even in GR for non-integrable $2+2$ decompositions, see details in [8, 9, 10]. This allows us to elaborate the concept of Einstein-Finsler gravity (EFG) gravity theory as an extension of GR to (co) tangent Lorentz manifolds with some well defined metric compatible Finsler connections (for instance, the Cartan and/or the canonical distinguished ones). For this class of theories, we do not need to introduce new definitions of Finsler space like in [6]; our Finsler constructions are dynamically derived on tangent spaces to Lorentz manifolds.

An important property of EFG field equations is that they can be integrated in very general diagonal and generic off-diagonal forms. Using certain classes of Finsler brane solutions constructed in [7] (in this letter, we work with the canonical distinguished connection instead of the Cartan and/or normal ones), we proved in explicit form that super-luminal effects for neutrinos can be explained by trapping from the Finsler spacetimes in the bulk to to real Minkowski and Einstein spacetimes. For such constructions, we still keep the standard paradigm of GR and SR but with some small modifications to include well-defined Finsler like connections for the bulk (co) tangent Lorentz bundles.

The challenge to reconcile theoretical results on dark energy and dark matter a with absence of superluminal propagation for supernova neutrinos still exists. To solve this problem following our approach is necessary to consider exact solutions in EFG theories for locally anisotropic and generic 
off-diagonal gravitational and Yang-Mills-Higgs-Diract fields interactions. Such solutions seem to be similar to those constructed in extra dimension, metric-affine and/or noncommutative theories [13].

Finally, we note that EFG theories can be quantized using deformation, A-brane and two connection formalism [11]. This provides us with new methods which allow us to study possible quantum gravitational and matter fields superluminal effects.

Acknowledgments. The research for this paper is partially supported by the Program IDEI, PN-II-ID-PCE-2011-3-0256. I'm grateful for for-

mer collaboration and/or important discussions/correspondence relevant to this paper to P. Stavrinos, N. Mavromatos, C. Lämmerzahl, V. Perlick, S. Odintsov, and C. Castro.

\section{References}

[1] OPERA Collaboration, arXiv: 1109.4897

[2] G. Amelino-Camelia, G. Gubitosi, N. Loret, F. Mercati, G. Rosati, P. Lipari, arXiv: 1109.5172; D. Fargion and D. D'Armeniento, arXiv: 1109.5368; J. Ciborowski, J. Rembielinski, arXiv: 1109.5599; K. Svozil, arXiv: 1109.5411; J. Alexandre, arXiv: 1109.5629; F. R. Klinkhamer, arXiv: 1109.5671; G. F. Giudice, S. Sibiryakov, A. Strumia, arXiv: 1109.5682; G. Dvali, A. Vikman, arXiv: 1109.5685; S. S. Gubser, arXiv: 1109.5687; P. Wang, H. Wu, H. Yang, arXiv: 1110.0449; J. Franklin, arXiv: 1110.0234; J. Alexandre, J. Ellis and N. Mavromatos, Phys. Lett. B706 (2012) 456, arXiv: 1109.6296; C. Castro, Found. Physics 42 (2012) 1135

[3] S. Vacaru, Ann. Phys. (NY) 256 (1997) 39; S. Vacaru, Nucl. Phys. B 434 (1997) 590; G. Amelino-Camelia, J. R. Ellis, N. E. Mavromatos, D. V. Nanopoulos, Int. J. Mod. Phys. A 12 (1997) 607; V. Perlick, Gen. Rel. Grav. 38 (2006) 365; F. Girelli, S. Liberati and L. Sindoni, Phys. Rev. D 75 (2007) 064015; P. C. Stavrinos, A. P. Kouretsis, M. Stathakopoulos, Gen. Rel. Grav. 40 (2008) 1403; A. P. Kouretsis, M. Stathakopoulos and P. C. Stavrinos, Phys. Rev. D 79 104011; S. Vacaru, J. Math. Phys. 50 (2009) 073503; S. Vacaru, arXiv: 1010.5457; F. P. Schuller, C. Witte, M. N. R. Wohlfarth, Ann. Phys. 325 (2010) 1853; N. E. Mavromatos, S. Sarkar, A. Vergou, Phys. Lett. B 696 (2011) 300; C. Castro, Int. J. Mod. Phys. A 26 (2011) 3653

[4] S. Vacaru, Class. Quant. Grav. 27 (2010) 105003

[5] C. Lämmerzahl, D. Lorek and H. Dittus, Gen. Rel. Grav. 41 (2009) 1345 
[6] C. Pfeifer, M. N. R. Wohlfarth, arXiv: 1109.6005; Phys. Rev. D 84 (2011) 044039

[7] S. Vacaru, Class. Quant. Grav. 28 (2011) 215001

[8] S. Vacaru, J. Phys. Conf. Ser. 314 (2011) 012069, arXiv: 1012.148; arXiv: 1004.3007; Int. J. Geom. Methods. Mod. Phys. 5 (2008) 473

[9] S. Vacaru, Int. J. Geom. Methods. Mod. Phys. 4 (2007) 1285; 8 (2011) 9; Int. J. Theor. Phys. 49 (2010) 884; arXiv: 1108.2022

[10] S. Vacaru, Phys. Lett. B 690 (2010) 224

[11] S. Vacaru, J. Geom. Phys. 60 (2010) 1289; Int. J. Geom. Methods. Mod. Phys. 6 (2009) 873; 7 (2010) 713

[12] M. Gogberashvili, Int. J. Mod. Phys. D 11 (2002) 1635; M. Gogberashvili, Europhys. Lett. 49 (2000) 396; M. Gogberashvili, Gravitational trapping for extended extra dimension, Int. J. Mod. Phys. D 11 (2002) 1639-1642; L. Randall and R. Sundrum, Phys. Rev. Lett. 83 (1999) 3370; L. Randall and R. Sundrum, Phys. Rev. Lett. 83 (1999) 4690; S. Vacaru and D. Singleton, Class. Quant. Grav. 19 (2002) 2793; P. Midodashvili, arXiv: hep-th/0308051; M. Gogberashvili and P. Midodashvili, Europhys. Lett. 61 (2003) 308; M. Gogberashvili and D. Singleton, Phys. Rev. D 69 (2004) 026004; D. Singleton, Phys. Rev. D 70 (2004) 065013; A. A. Colley, S. Hervik, Class. Quant. Grav. 21 (2004) 5759; C. G. Boehmer, G. De Risi, T. Harko, F. S. N. Lobo, Class. Quant. Grav. 27 (2010) 18501

[13] S. Vacaru and F. C. Popa, Class. Quant. Gravity, 18 (2001) 4921; S. Vacaru, D. Singleton, V. Botan and D. Dotenco, Phys. Lett. B 519 (2001) 249; S. Vacaru and D. Singleton, Class. Quant. Grav. 19 (2002) 3583

[14] G. Y. Bogoslovsky, Nuovo. Cim. B77 (1983) 181; G. S. Asanov, Finsler Geometry, Relativity and Gauge Theories (D. Reidel Publishing, Dordrecht, 1985); R. Miron and M. Anastasiei, The Geometry of Lagrange Spaces: Theory and Applications, FTPH no. 59 (Kluwer Academic Publishers, Dordrecht, Boston, London, 1994); D. Bao, S. S. Chern and Z. Shen, An Introduction to RiemannFinsler Geometry (Springer, NY, 2000); G. Bogoslovsky and H. F. Goenner, Plys. Lett. A323 (2004) 40; G. W. Gibbons, J. Gomis, C. N. Pope, Phys. Rev. D 76 (2007) 081701

[15] P. Adamson et al. [MINOS Collaboration], Phys. Rev. D76 (2007) 072005; G. R. Kalbfleisch, N. Baggett, E. C. Fowler and J. Alspector, Phys. Rev. Lett. 43 (1979) 1361 